\title{
Occurrence of polar stratospheric clouds at Kiruna
}

\author{
C.-F. Enell ${ }^{1}$, Å. Steen ${ }^{1}$, T. Wagner ${ }^{2}$, U. Frieß ${ }^{2}$, K. Pfeilsticker ${ }^{2}$, U. Platt ${ }^{2}$, K.-H. Fricke ${ }^{3}$ \\ ${ }^{1}$ Swedish Institute of Space Physics, P.O. Box 812, SE-98128 Kiruna, Sweden \\ ${ }^{2}$ Institute of Environmental Physics, Heidelberg University, INF 229, D-69120 Heidelberg, Germany \\ ${ }^{3}$ Institute of Physics, Bonn University, Nussallee 12, D-53115 Bonn, Germany
}

Received: 7 May 1999 / Revised: 2 July 1999 / Accepted: 7 July 1999

\begin{abstract}
Polar stratospheric clouds (PSCs) are often observed in the Kiruna region in northern Sweden, east of the Scandinavian mountain range, during wintertime. PSC occurrence can be detected by ground-based optical instruments. Most of these require clear tropospheric weather. By applying the zenith-sky colour index technique, which works under most weather conditions, the data availability can be extended. The observations suggest that PSC events, especially of type II (water PSCs) may indeed more common than predicted by synoptic models, which is expected because of the frequent presence of mountain-induced leewaves. However, it will be of importance to increase the density of independent observations.
\end{abstract}

Key words. Atmospheric composition and structure (aerosols and particles, cloud physics and chemistry) · Meteorology and atmospheric dynamics (mesoscale meteorology)

\section{Introduction}

It is well known that Polar Stratospheric Clouds (PSCs), which develop in the winter polar vortex at altitudes of typically $25 \mathrm{~km}$, are of major importance in stratospheric chemistry (Solomon et al., 1986). They may also be important as an indicator of climate change, but the correlation between the frequency of PSC observations and stratospheric cooling is still not well known. This is in itself a good reason for improving statistics on PSC occurrence.

Normally PSC presence is inferred from modelled synoptic temperatures, such as provided by the European Centre for Medium-Range Weather Forecasts

Correspondence to: C.-F. Enell, e-mail: carl-fredrik.enell@irf.se
(ECMWF). That method does not account for PSC formation by local cooling in mountain-induced waves, which can be observed in certain areas close to mountain ridges, such as the Kiruna region in northern Sweden.

It has been proposed by Carslaw et al. (1998) that these wave-induced PSCs may be a major cause of the discrepancies between observed and modelled ozone loss. However, their importance is still the object of much controversy. An experimental climatology of actual PSC observations is therefore desirable, which means that the density of such observations must be increased. A number of optical remote-sensing instruments suitable for that purpose are at present in operation around the Scandinavian mountain range, both active (lidars) and passive (spectrometers and cameras).

The final aim may be PSC mapping using satellite data (Garcia et al., 1995; Meerkötter, 1995). This procedure will require calibration against ground-based instruments.

\section{Methods}

\subsection{Zenith-sky colour index}

The zenith-sky colour index method was proposed as a way of obtaining PSC statistics in all tropospheric weather conditions and has been described by Sarkissian et al. (1991). In principle the method is very simple and any spectrometer or combination of filter photometers covering a suitable interval in the visible spectral range can be used. A short outline of the technique follows. For further details the reader should consult Sarkissian et al. (1991) and the references therein.

Taking the integrated intensities $I\left(\lambda_{1}\right)$ and $I\left(\lambda_{2}\right)$ over two wavelength intervals around $\lambda_{1}$ and $\lambda_{2}$ a colour index can be defined as:

$\mathrm{CI}=\frac{I\left(\lambda_{1}\right)}{I\left(\lambda_{2}\right)}$ 
This colour index varies with the solar zenith angle (SZA), tropospheric cloud cover, varying absorptions, aerosols, etc.* In general we will write $\mathrm{CI}=\mathrm{CI}(\mathrm{SZA})$. When PSCs or other layers of enhanced scattering, such as tropospheric clouds, are present, the SZA dependence of the colour index will change. In general reddenings (i.e. increased intensities at longer wavelengths compared to the case of pure clear-sky Rayleigh scattering) will be observed in certain SZA intervals.

Since December 1996, a zenith-looking UV/visible spectrometer system is in operation at the Swedish Institute of Space Physics (in Swedish Institutet för Rymdfysik, abbreviated IRF), $67.8^{\circ} \mathrm{N}, 20.4^{\circ} \mathrm{E}, 418 \mathrm{~m}$ above sea level. The instrument is used on a routine basis for retrieval of total column densities of the species ozone, $\mathrm{NO}_{2}, \mathrm{BrO}$ and $\mathrm{OClO}$ using the Differential Optical Absorption Spectroscopy (DOAS) method (Platt, 1994).

Wavelengths (in our case, integrated over 5 out of 1024 instrument channels at the ends of the spectral intervals covered by the instruments) were selected according to Table 1 . These wavelengths should preferrably be selected outside regions of large varying absorptions. It should be emphasized that these colour indices are relative quantities, since the characteristics of the filters used, spectral response of the detectors, etc, need not necessarily be known.

\subsection{Detection of PSCs}

For experimental validation of the method, four ideal type cases according to Table 2 were identified from records of visual meteorological observations. Colour indices (Eq. 1) were then calculated for the solar zenith angle (SZA) intervals $85^{\circ}<\mathrm{SZA}<96^{\circ}$ over the sunset periods of those days (Fig. 1).

2.2.1 The non-PSC cases. The clear-day curve shows exactly the behaviour of $C I$ vs $S Z A$ that can be expected from our everyday experience of the appearance of the evening sky. After sunset the sky reddens slightly as expected due to Rayleigh scattering along the increasing optical path the sunlight has to traverse. After $\mathrm{SZA} \approx 92^{\circ}$ a blueing sets in, which at first may seem unexpected although it agrees with visual observation. This long-known phenomenon is due to ozone Chappuis absorption, as explained e.g. by Hulburt (1953), and possibly aerosol scattering. A comprehensive description

Table 1. Wavelengths used for calculation of the CI

\begin{tabular}{lll}
\hline & Present work & Sarkissian et al. \\
\hline$\lambda_{1}$ & $680 \mathrm{~nm}$ & $550 \mathrm{~nm}$ \\
$\lambda_{2}$ & $385 \mathrm{~nm}$ & $350 \mathrm{~nm}$ \\
\hline
\end{tabular}

* Colour is a physiological rather than a physical quantity, but an increase of the colour index as defined here will indeed correspond to a reddening of the zenith sky.
Table 2. Days (YYMMDD) of ideal observational conditions

\begin{tabular}{lll}
\hline & Clear sky & Cloudy sky \\
\hline No PSC & 980302 & 980324 \\
PSC & 970116 & 970121 \\
\hline
\end{tabular}

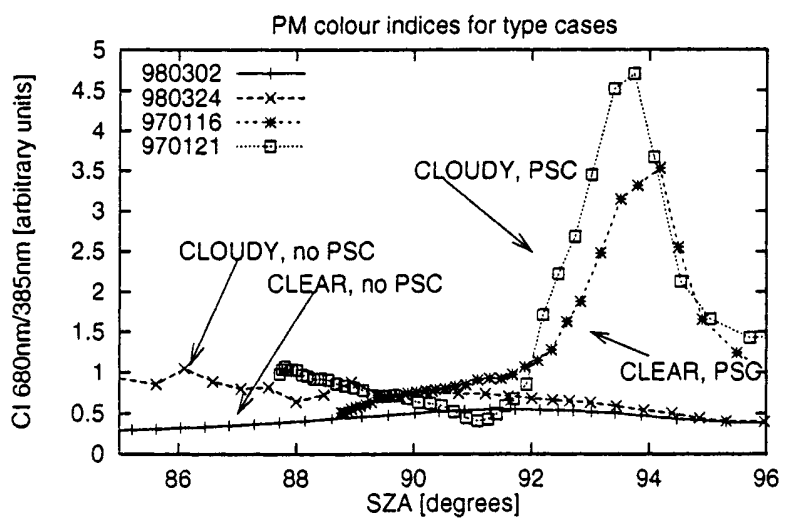

Fig. 1. Colour index development during twilight in the four type cases of Table 2

of twilight sky phenomena, including colour index variation, can be found in Rozenberg (1966).

For the cloudy days the sky is reddened to an extent varying with the cloud cover during daytime. During twilight the colour index falls off towards its clear-sky values as tropospheric clouds fall into darkness.

2.2.2 The PSC cases. For the days with PSC presence the CI shows approximately the same behaviour as on nonPSC days for $S Z A<90^{\circ}$. The clear-sky case shows a positive slope (slow reddening) whereas the cloudy-sky case shows a slow blueing up to a solar zenith angle of $\approx 92^{\circ}$. This SZA corresponds to an altitude of maximal scattering (Solomon et al., 1987) at typical PSC altitudes. There, a significant reddening occurs (CI up to one order of magnitude larger than in the non-PSC cases).

Two effects may contribute to this reddening: enhanced scattering of sunlight reddened by Rayleigh extinction along the long atmospheric optical path at twilight, and the significantly different wavelength dependence in scattering by large particles as opposed to the $1 / \lambda^{4}$ dependence of scattering by air molecules. This mechanism is clearly not strongly affected by clouds in the (during twilight relatively dark) troposphere between the instrument and the PSC layers.

A further understanding of the process can be gained by multiple-scattering Monte-Carlo radiative transfer modelling, which is work in progress.

2.2.3 Uncertainties. Cases other than the ideal ones described above are important to examine. For instance, the results of Fig. 2 are of special interest. They were obtained using data from days that, according to the 


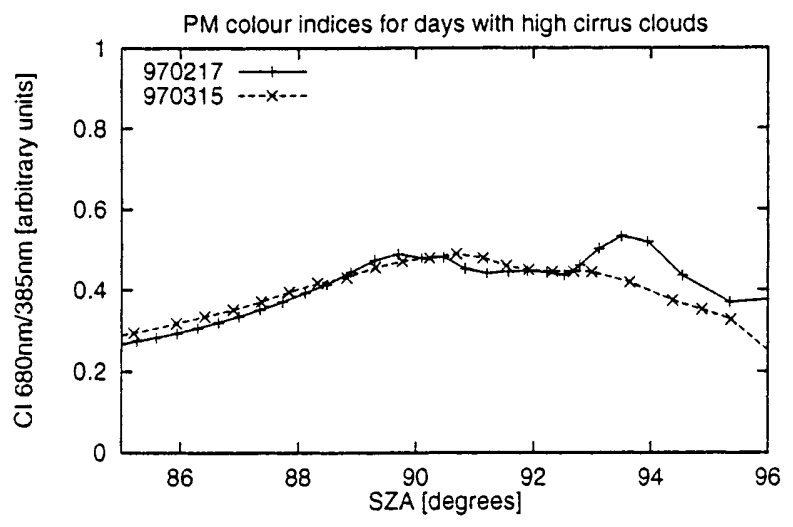

Fig. 2. Colour index development during days with high cirrus clouds

limited visual observations available, were mainly clear but with high cirrus clouds present. Between 89 and $94^{\circ}$ SZA in this graph, some features that may be interpreted as a slight reddening occur. This is also consistent with some observations by Sarkissian et al. (1991). Thus, for this reason as well as for changes caused by varying ozone concentrations, small reddenings have to be considered insignificant.

2.2.4 Interpretation of the CI curves. It can be seen that the important signature of PSCs is a large positive (relative to the non-PSC cases) CI slope during twilight. This quantity is proportional to the ratio of the CI at a certain SZA $\left(>90^{\circ}\right)$ to the CI at $S Z A=90^{\circ}$, a ratio that is usually called normalized colour index.

It can be argued that it may be important to correct the measured spectra for all known absorptions and other effects not caused by PSC scattering. This was emphasized in Sarkissian et al. (1991). However, our intention here is to find a simplified method suitable for use with all kinds of instrumentation, such as spectrometers, filter photometers, etc, where the column amounts of ozone and other trace species are not easily calculated.

Different features, such as impact of tropospheric clouds, distant PSCs and varying absorptions, should instead be recognized by a careful classification of CI plots. This, however, requires information on the overall meteorological and PSC situation by means of daylight all-sky imaging, visual observations, etc, which are not available at present. The development of such observations and classification methods is an aim of our future work.

2.2.5 Data availability. As was demonstrated above, it is possible to detect polar stratospheric clouds when the sun is is in a certain zenith angle interval. A reasonable assumption is to take $92^{\circ}<S Z A<95^{\circ}$ as a usable range. The local time of these SZA intervals - calculated for the geographical location of IRF using the graphical ephemeris software XEphem - vs date is plotted in Fig. 3. The duration of this twilight interval is on the order of $30 \mathrm{~min}$. Mountain-induced PSCs develop over timescales of hours and will thus be undetectable during most of the day. Therefore the method may underestimate the occurrence of such events.

\subsection{Visual observations}

2.3.1 Availability of observations. Like the zenith-sky measurements, visual observations are limited by requirements on solar zenith angle and viewing angle intervals, since the distinct iridescent appearance (or deep red colour) that characterizes some stratospheric clouds (most probably ice PSCs, although PSC type I have with high certainty been observed at the site as "purple twilights") is only visible at certain scattering angles. If these conditions are not met, it is not possible to identify stratospheric clouds by monostatic observations alone, since altitude information is often required to distinguish them from high tropospheric clouds such as cirrus. However, the most severe limitation of direct visual observations is tropospheric clouds.

\subsection{Lidar observations}

A lidar system is operated on campaign basis at the nearby site of Esrange. The distance to the location of the UV-visible spectrometer is approximately $30 \mathrm{~km}$ so the two instruments are likely to observe different air masses. However, they are in the vicinity of the same gridpoint of the synoptic ECMWF model, so they can be used in order to study the importance of mesoscale deviations.

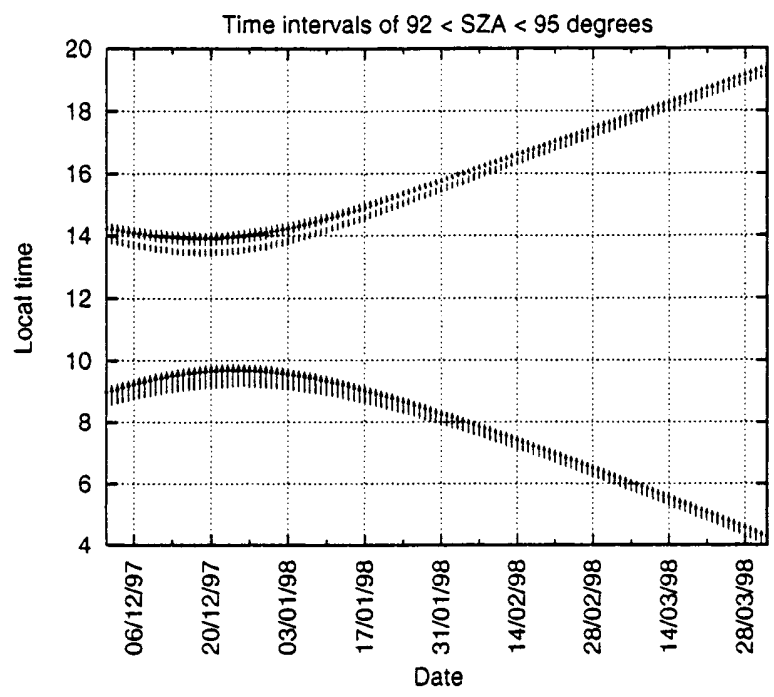

Fig. 3. Time intervals (marked by arrows) of $92^{\circ}<\mathrm{SZA}<95^{\circ}$ calculated for Kiruna $\left(68^{\circ} \mathrm{N}, 20^{\circ} \mathrm{E}\right)$ 
2.4.1 Data availability. Since the lidar is an active instrument, observations are not limited to sunrise and sunset but are most conveniently performed at nighttime. Thus we cannot expect the two instruments to measure during exactly the same events. Rather, visual, CI and lidar observations complement each other to provide a coverage of mesoscale and synoptic PSC events within the Kiruna area.

\section{Experimental results}

\subsection{Visual observations}

Records of visual PSC observations (most probably isolated PSC type II structures) were kept during the European leewave campaigns (Fricke et al., 1998) in the winters of 1996-1997 and 1997-1998. During other periods visual records cannot be considered complete.

\subsection{Colour indices}

Colour indices according to Eq. (1) and Table 1 were evaluated for the winter seasons of 1996-1997 and 19971998. The ratios $C I_{\text {norm }}$ of observed colour indices to the interpolated values at $\mathrm{SZA}=90^{\circ}$, i.e.

$\mathrm{CI}_{\text {norm }}=\frac{\mathrm{CI}(\mathrm{SZA})}{\mathrm{CI}\left(90^{\circ}\right)}$

were formed and averaged over the interval $93.5^{\circ}<\mathrm{SZA}<94^{\circ}$, where the CI generally shows its maximum when PSCs are present, for each sunrise and sunset (Fig. 4). Figure 5 shows histograms of these values for the two winters. In Fig. 6 the days with obvious PSC presence have been removed. This suggests a PSC threshold value of $\mathrm{CI}_{\text {norm }} \approx 0.8$.

\subsection{Lidar observations}

The lidar detects atmospheric backscatter at $532 \mathrm{~nm}$ in polarisations parallel and perpendicular to the emitted beam. The backscatter ratio and depolarisation depend on the size and shape of the particles. Thus the lidar is capable of distinction between PSC type I - saturated ternary solution (STS)/nitric acid tetrahydrate (NAT), and PSC type II - water ice (See Table 3).

\subsection{Summary of observations}

All detections of PSCs by any of the methods $\left(\mathrm{CI}_{\text {norm }}>0.8\right.$, lidar or visual observation $)$ are collected in Figs. 7 and 8. Lidar observations are divided according to polarisation - parallel only or depolarisation (signal in the cross-polarised channel). Included is also modelled synoptic incidence of PSC type I and II, respectively (see sect. 4).

The CI detections are by far more comprehensive because of the insensitivity to tropospheric clouds and
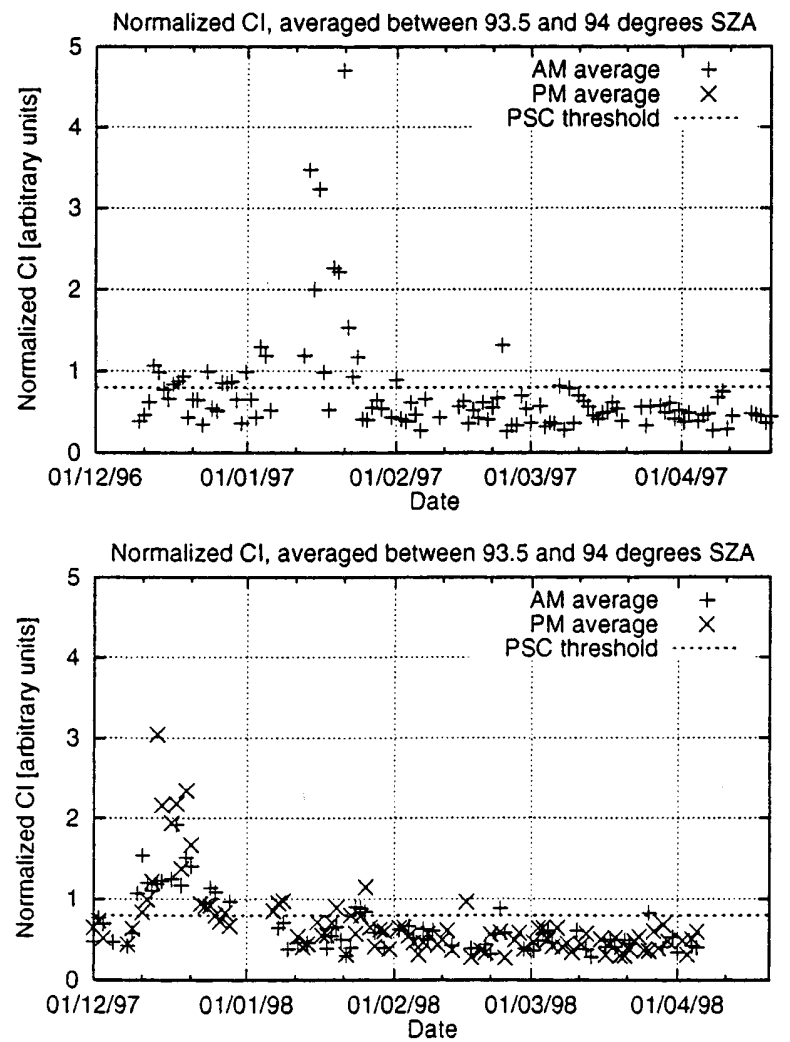

Fig. 4. Time series of colour index ratios $\left[\mathrm{CI}_{\text {norm }}=\mathrm{CI}(\mathrm{SZA}) /\right.$ $\left.\mathrm{CI}\left(90^{\circ}\right)\right]$
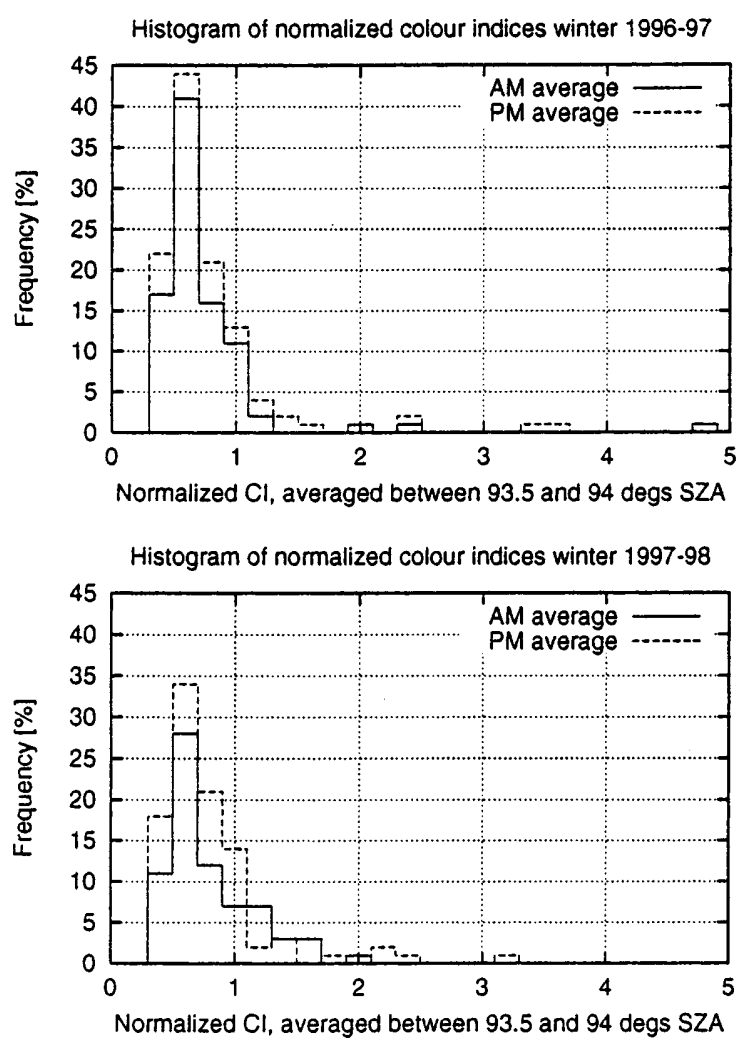

Fig. 5. Histograms of colour index ratios $\left[\mathrm{CI}_{\text {norm }}=\mathrm{CI}(\mathrm{SZA}) /\right.$ $\left.\mathrm{CI}\left(90^{\circ}\right)\right]$ 


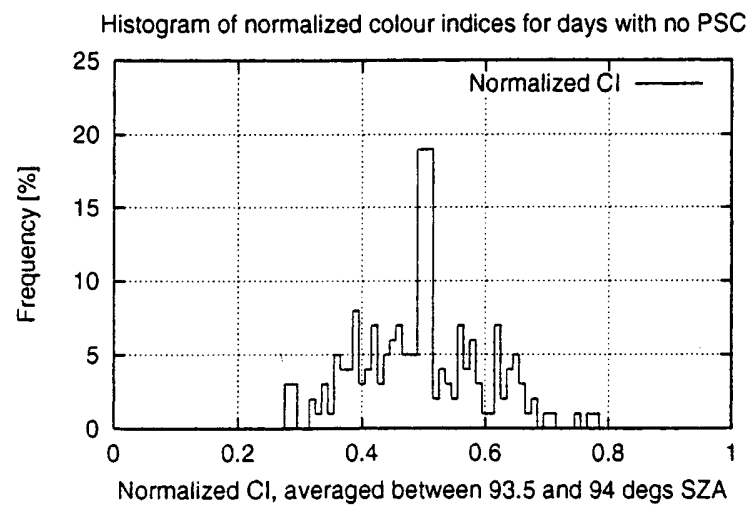

Fig. 6. Histograms of colour index ratios $\left[\mathrm{CI}_{\text {norm }}=\mathrm{CI}(\mathrm{SZA}) /\right.$ $\mathrm{CI}\left(90^{\circ}\right)$ ]; data from days with obvious PSC observations are removed

Table 3. Interpretation of lidar data

\begin{tabular}{ll}
\hline Observation & Composition \\
\hline Low backscatter ratio, parallel & STS \\
Low backscatter ratio, depolarisation & NAT \\
High backscatter ratio, depolarisation & Ice \\
\hline
\end{tabular}

the automation of the measurements. Missing lidar or visual observations mean either cloudy weather, no PSC detection or no observations.

From these data it can be concluded that significant CI reddenings do correspond to incidence of PSC type I or II. The largest reddenings were actually coincident with synoptic presence of PSC type II as deduced from lidar observations and calculated temperatures.

Table 4 shows the frequency of PSC presence as inferred from $\mathrm{CI}$ reddenings. The total numbers of twilight measurements (AM and PM) available are also included. The significantly less number of AM measurements is due to frequent power failures, network

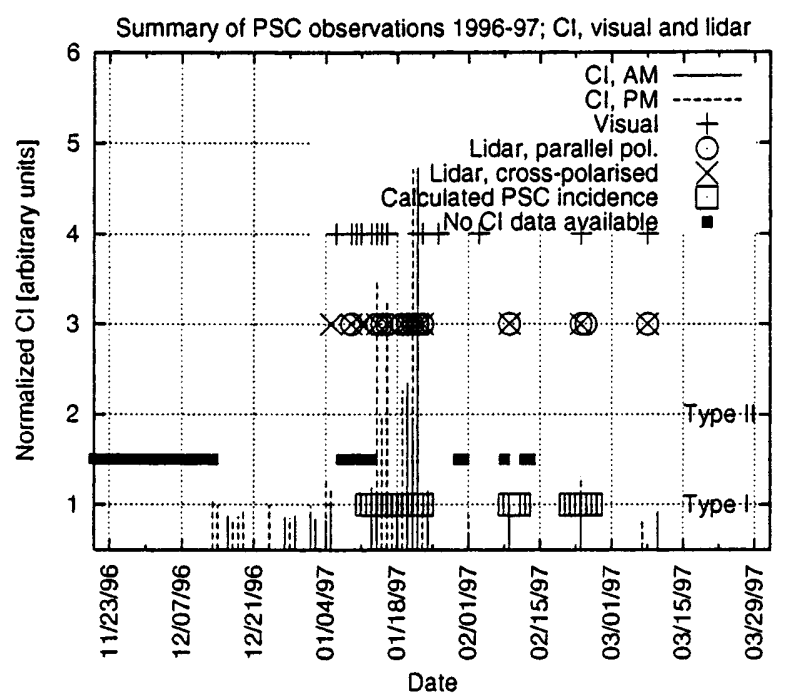

Fig. 7. Observations of PSCs in the winter of 1996-1997

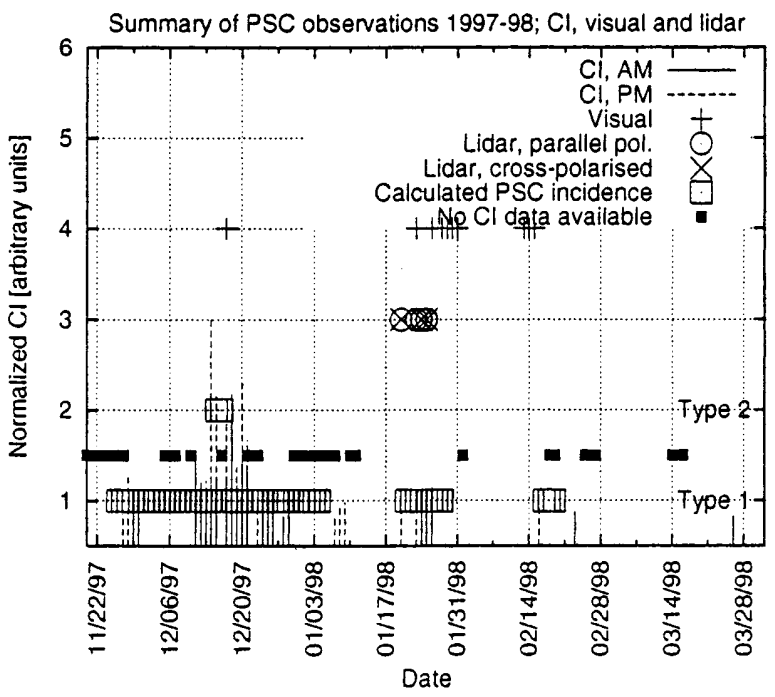

Fig. 8. Observations of PSCs in the winter of 1997-1998

shutdowns, etc., during nights. On such occasions the instrument has to be restarted manually in the morning.

\section{Synoptic PSC presence}

As outlined in sect. 1 the role of mesoscale PSC events (as opposed to synoptic PSC and aerosol presence) in stratospheric chemistry is still controversial. Some authors (Carslaw et al., 1998) believe that such events explain the excess ozone loss that has until now not been possible to account for by chemistry/transport (CTM) models. A starting point in understanding this can be provided by comparing the above observations with synoptic models.

One-point-per-day calculations of PSC incidence on the isentropic levels $475 \mathrm{~K}, 550 \mathrm{~K}$, and $675 \mathrm{~K}$ (as inferred from ECMWF temperatures) were retrieved using the program isen $t s$ available at NILU (Braathen et al., 1998). Calculated PSC incidence on any of these altitudes is included in the Figs. 7 and 8.

A constant water vapour VMR of $4.6 \cdot 10^{-6}$ and a LIMS NAT profile from January 1979 are used in these calculations. However, even large changes in these parameters typically yield differences in NAT and $\mathrm{H}_{2} \mathrm{O}$ condensation temperatures on the order of 1, K (Hanson and Mauersberger, 1988; Marti and Mauersberger 1993), whereas leewave activity easily causes temperature changes on the order of $10 \mathrm{~K}$, so these assumptions introduce no major error. However, this model fails to

Table 4. Numbers of twilights with PSC incidence (out of total number of measurements) as deduced from CI reddenings for the winters of 1996-1997 and 1997-1998

\begin{tabular}{lllcl}
\hline & CI AM & CI PM & Total AM & Total PM \\
\hline Winter 1996-1997 & 16 & 26 & 90 & 113 \\
Winter 1997-1998 & 21 & 25 & 72 & 101 \\
Total & 37 & 51 & 162 & 214 \\
\hline
\end{tabular}


Table 5. Numbers of days with modelled PSC incidence for the winters of 1996-1997 and 1997-1998

\begin{tabular}{llll}
\hline & PSC type I & PSC type II & Total days \\
\hline Winter 1996-1997 & 20 & 0 & 132 \\
Winter 1997-1998 & 45 & 2 & 135 \\
Total & 65 & 2 & 267 \\
\hline
\end{tabular}

predict the observed synoptic PSC type II occurrences (which persisted for several days) in January 1997.

In Table 5, the total number of days with calculated PSC incidence thus obtained follows.

\section{Discussion}

In general there seems to be a good agreement between observed CI reddenings and calculated synoptic PSC type I occurrence. During the winter of 1996-1997 the frequency of observed CI reddenings (according to Table 4: $18 \%$ of all sunrises and $23 \%$ of all sunsets) was slightly higher than that of modelled PSC incidence (15\% of all days according to Table 5). In the winter of 1997-1998 the frequency of observed PSC events was in fact lower than the frequency of calculated events but this can be explained by the fact that there are long periods of missing CI data coincident with cold periods during that winter.

What is clearly underestimated, however, by the synoptic temperature approach to PSC incidence modelling is the occurrence of PSC type II. Very large reddenings, such as the cases of January and December 1997, were coincident with visual observations of PSC type II. The model predicts mainly PSC type I on those occasions.

Except for very rare events, such as the aforementioned case of 16-19 January 1997, PSC type II are mainly observed as discrete structures developing in mountain waves. This is naturally more likely to happen if synoptic conditions allow the presence of PSC type I. It can nevertheless be concluded that both the observations (for reasons explained in sects. 2.2.3 and 2.2.5) and the modelling underestimate the occurrence of these events, but observations suggest that they are indeed more common than predicted by synoptic modelling.

\section{Outlook}

Future work in this field will include a comparison between stations at different locations with respect to the Scandinavian mountain range (Andøya, Norway; Kiruna, and Sodankylä, Finland) and also development of a denser network of photometers and imagers, which will improve the coverage of mesoscale PSCs. This can be expected to help resolve the question of the importance of mesoscale PSCs and will also serve as ground-truth for PSC mapping from satellites.

Acknowledgements. The authors thank their colleagues for fruitful discussions and Alain Sarkissian for comments on the method. The work was partly supported by MRI, the Environment and Space Research Institute in Kiruna. ECMWF temperature data and PSC incidence calculations were obtained from the Norwegian Institute of Air Research (NILU). Since February 1999 the colour index data are available in real time on the WWW. See http://www.irf.se/ fredrik.

Topical Editor F. Vial thanks K. Kreher and A. Sarkissian for their help in evaluating this paper.

\section{References}

Braathen, G., L. Aanesland, and B. Bojkov, NADIR News, Norwegian Institute of Air Research, 1998.

Carslaw, K. S., M. Wirth, A. Tsias, B. P. Luo, A. Dörnbrack, M. Leutbecher, H. Volkert, W. Renger, J. T. Bacmeister, E. Reimers, and T. Peter, Increased stratospheric ozone depletion due to mountain-induced atmospheric waves, Nature, 391, 675678, 1998.

Fricke, K.-H., K. P. Müller, M. Serwazi, J. Reichardt, S. Kirkwood, A. Steen, P. Hoffman, H. Mehrtens, A. Hauchecorne, F. Fierli, U.-P. Hoppe, and G. Hansen, Wind generated polar stratospheric clouds: The case of January 16, 1997 above Northern Scandinavia, in Polar stratospheric ozone 1997, Proceedings of the fourth European symposium 22 to 26 September 1997, Schliersee, Bavaria, Germany, pp. 127-130, European Commission, 1998.

Garcia, O., K. Pagan, P. Foschi, S. Gaines, and S. Hipskind, Detection of polar stratospheric clouds over Antarctica using AVHRR images obtained at Palmer station during August 1992, Polar Record., 177, 211-226, 1995.

Hanson, D., and K. Mauersberger, Laboratory studies of the nitric acid trihydrate Implications for the south polar stratosphere, Geophys. Res. Lett., 15, 855-858, 1988.

Hulburt, E., Explanation of the brightness and color of the sky, particularly the twilight sky. J. Opt. Soc. Am., 43, 113-118, 1953.

Marti, J., and K. Mauersberger, A survey and new measurements of ice vapor pressure at temperatures between 170 and $250 \mathrm{~K}$. Geophys. Res. Lett., 20, 363-366, 1993.

Meerkötter, R., Detection of polar stratospheric clouds with ERS2/GOME data, Ann. Geophys., 13, 395-405, 1995.

Platt, U., Differential optical absorption spectroscopy, in Air Monitoring by Spectroscopic Techniques, chap. 2. J Wiley, 1994.

Rozenberg, G. V., Twilight, Plenum Press, New York 1966.

Sarkissian, A., J.-P. Pommereau, and F. Goutail, Identification of polar stratospheric clouds from the ground by visible spectrometry, Geophys. Res. Lett. 18, 779-782, 1991.

Solomon, S., R. R. Garcia, F. S. Rowland, and D. J. Wuebbles, On the depletion of Antarctic ozone, Nature, 321, 755-758, 1986.

Solomon, S., A. L. Schmeltekopf, and R. W. Sanders, On the interpretation of zenith sky absorption measurements, J. Geophys. Res. 7, 8311-8319, 1987. 